\title{
MOOC (Massive Online Open Courses) como método-plataforma educativa en el ámbito universitario ${ }^{1}$
}

\author{
Jorge CALDERA-SERRANO \\ Departamento de Información y Comunicación \\ Universidad de Extremadura \\ jcalser@alcazaba.unex.es \\ José-Antonio LEÓN-MORENO \\ Consultor T.I.C. \\ yo@jaleon.es
}

Recibido: 25/06/2015

Aceptado: 21/09/2015

\begin{abstract}
RESUMEN
Se describen y analizan los MOOCs (Massive Online Open Course) como método de difusión de información y documentación en los ámbitos educativos universitarios. Para ello se describe que son los MOOCs, mostrando su pujanza y su reciente aparición y desarrollo, y describiendo las muchas potencialidades y posibilidades que plantean, al igual que se describen los principales problemas. El desarrollo y utilidad de los MOOCs se ha planteado especialmente en el ámbito universitario, siendo utilizado como mecanismo para facilitar cursos en línea con el fin de difundir conocimiento científico y como método de marketing y financiación en las instituciones de educación superior.
\end{abstract}

Palabras clave: MOOC, Educación Universitaria, Enseñanzas en línea, Marketing universitario, Bibliotecas universitarias.

\section{MOOC (Massive Online Open Courses) as a platform method-learning in the university}

\begin{abstract}
It describes and analyzes the MOOCs (Massive Online Open Course) as a method of disseminating information and documentation in university educational environments. We describe which are the MOOCs, showing its strength and its recent emergence and development, and describing the many potentials and possibilities posed, like the main problems are described. The development and utility of MOOCs has arisen especially in the university, being used as a mechanism to provide online courses to disseminate scientific knowledge and method of marketing and finance in higher education institutions.

Keywords: MOOC, High Education, E-learning, University Marketing, University Library.
\end{abstract}

\footnotetext{
${ }^{1}$ Este trabajo ha sido financiado por la Junta de Extremadura (Consejería de Educación, Ciencia y Tecnología) y el Fondo Social Europeo dentro del plan de apoyo a las actuaciones de los Grupos de Investigación inscritos en el catálogo de la Junta de Extremadura. GR10019.
} 


\section{Referencia normalizada}

Caldera-Serrano, J.; León-Moreno, J.A. (2015). “MOOC (Massive Online Open Courses) como métodoplataforma educativa en el ámbito universitario”. Documentación de las Ciencias de la Información, Vol. 38: páginas 301-310.

\section{INTRODUCCIÓN}

Desde su teorización por George Siemens y Stephen Downes (2008) hasta ahora, los denominados MOOC's, acrónimo en inglés de Massive Online Open Courses (o Cursos Abiertos Online y Masivos), han causado un efecto positivo muy amplio y de impacto en el mundo de la educación universitaria, ya que son un claro ejemplo de formación a distancia planificados para alcanzar un número ilimitado de usuarios, debido a su carácter abierto, participativo y con una metodología basada en el conocimiento.

Para que la enseñanza a distancia pueda ser considerada MOOC debe cumplir los siguientes requisitos según la Wikipedia (2013):

- Ser un curso: Debe contar con una estructura orientada al aprendizaje, que conlleve una serie de pruebas y de evaluaciones para acreditar el conocimiento adquirido.

- Tener carácter masivo: El número de posibles matriculados es ilimitado tanto por su número como por su lugar de matriculación, no se circunscribe territorialmente. Su alcance es global.

- En línea: El curso es a distancia e Internet es el medio de comunicación.

- Abierto: Los materiales son accesibles de forma gratuita y de acceso libre en Internet.

Los MOOC están siendo ampliamente aceptados por la comunidad universitaria, incluso por las universidades más prestigiosas, aquellas que presencialmente cuentan con un número de alumnos importante y sobre todo con un amplio prestigio y bien posicionado en rankings internacionales. Entonces cabe preguntarse el porqué de ofertar formación MOOC's. Que Harvard, Stanford, o el Massachusetts Institute of Technology (MIT) ofrezcan sus propios cursos mediante la modalidad MOOC puede ser visto como una forma de hacerse competencia a sí mismos, una forma de ampliar su catálogo formativo, o de perjudicarse a sí mismos por crear una "línea blanca" de la educación.

Sin embargo, la situación puede ser toda la contraria: puede ser una forma de hacerse publicidad en el "nuevo orden mundial", puede ser una forma de captar a los mejores estudiantes de todo el mundo y que posteriormente accedan a contratos de las grandes compañías que han invertido de forma indirecta en su formación al financiar los MOOC's, o incluso una forma viral de captar alumnos. 


\section{CONCEPTUALIZACIÓN}

Como se ha mencionado con anterioridad es unánime el situar su origen en el Curso sobre Conectivismo que organizaron Siemens y Downes en 2008. Si bien antes, podemos encontrar iniciativas como el Open Coruseware del MIT en el año 2001 o la Khan Academy cinco años después. Siendo precedentes, estos cursos carecían de dos de los componentes que se han revelado claves en los MOOC: su carácter masivo (Massive) y el hecho de que sean cursos reales con un profesor al frente (Courses). Pero, si el origen fue 2008, su popularización no llegó hasta el otoño de 2011 con el Curso sobre Inteligencia Artificial (The New York Time. Science, 2011) liderado por los profesores de computación de la Universidad de Standford, Peter Norvig y Sebastian Thrun.

Desde hace años, existe un movimiento denominado Cultura Libre (Wikipedia, 2013 b) que promulga la generalización de la cultura y que, poco a poco, va impregnando otros ámbitos de la vida como el software Libre que es su máxima expresión, además de otros elementos destacados tales como el Open Access y crowfunding (entre otros), los cuales conjugan un ecosistema donde la aparición de los MOOC's era inevitable derivado del surgimiento de varios factores tales como la conectividad, el pensamiento open, la demanda del acceso abierto a textos científicos, el open data, etc.

Una vez situado su contexto, hemos de conceptualizar que los MOOCs tienen una fundamentación epistemológica que sería el conectismo (Wikipedia, 2013, c) que se define como el efecto que la tecnología ha tenido sobre la manera en que actualmente vivimos, nos comunicamos, relacionamos y aprendemos.

Usaremos el término conectismo con el mismo valor que el término conectivismo, ya que su traducción literal y más correcta al español sería conectismo (raíz "conect-" y sufijo “-ismo”).

Downes (2011) señala que "Los MOOCs combinan el contenido abierto y la enseñanza abierta pero también son compatibles con la participación masiva, y esto sólo se logra mediante la adopción de una pedagogía y una estructura conectivista”.

El conectivismo para Siemens (2005) se ha presentado como una teoría que supera "las tres grandes teorías" sobre el aprendizaje: conductismo, cognitivismo y constructivismo. Afirma igualmente "que el conectivismo (...) es una teoría" que se caracteriza por considerar el aprendizaje como una extensión "del aprendizaje (ya existente), del conocimiento y de la comprensión a través de la extensión de una red personal".

Pero además de su conceptualización epistemológica, lo que hace que los MOOC's sea considerada como una de las tecnologías disruptivas de 2013 ha sido su vertiginosa irrupción avalados por los datos, pues plataformas como Udacity (http://www.udacity.com) ha captado 15 millones de dólares de inversión y 475.000 alumnos han cursado ya alguna materia. Coursera (http://www.coursera.org) es una corporación que engloba 33 universidades entre las más prestigiosas del mundo (Princenton, Stanford, Columbia) la cual ha tenido 7 millones de inscritos en sus cursos masivos abiertos en línea (MOOC) hasta el 18 de diciembre pasado de 2012, 
esto ha hecho que la propia universidad de Harvard incluyera los MOOC's como una de las tendencias disruptivas del 2013 (Harvard Business Review, 2013).

No obstante, pretendemos mostrar las características y detalles de esta irrupción tecnológica de este tipo de plataformas, así como aquellos items que por el contrario muestran que estas plataformas tienen mucho de marketing y de "sexy knowledge" (http://www.sexyknowledge.com), pero especialmente queremos destacar como puede ser una útil estrategia de las grandes Universidades que se están protegiendo ante el previsible estallido de la "burbuja de las universidades" ("higher college bubble”).

\subsection{Características de los MOOC's}

Se ha comentado cuál es la definición de un MOOC y qué se entiende por tal, pero ahora vamos a tratar de determinar cuáles son las características que hacen que un curso sea considerado MOOC.

Thrun y Norvig (2012) señalan que la metodología docente para los MOOC debe contar con los siguientes elementos:

- Vídeo Lecciones. Exposiciones magistrales del tutor grabadas en pequeños clips de vídeo de como máximo 15 minutos de duración, lo que constituye el método principal para comunicar los contenidos durante el curso y suelen tener una periodicidad semanal. Localizadas en la red y accesibles desde cualquier dispositivo pueden ser seguidas por los alumnos a su propio ritmo y no de manera secuencial.

- Cuestionarios. Son pruebas en línea que permiten al alumno demostrar su conocimiento sobre los temas que se está capacitando.

- Tareas. Ejercicios que están disponibles durante la semana y que han de completar de manera correcta, pues de su resolución dependerá la solvencia en el examen posterior.

- Exámenes. Son las calificaciones globales después de responder con mayor o menor fiabilidad las preguntas que sobre los conceptos expuestos durante la semana se han tratado en las tareas y cuestionarios. Los exámenes tratarán los mismos temas que en las evaluaciones anteriores pero de una manera más profusa.

- Foros y/o Tablones. Cuestiones que se plantean a los profesores de manera directa. Estas preguntas pueden ser visualizadas por el resto de los alumnos y votar por ellas. Los instructores responderán a las preguntas más votadas.

Los MOOC's son unas de las tecnologías disruptivas de este 2013, pero su evolución a corto plazo la convertirá en una tecnología muy diferente a la actual.

La configuración de la Educación Superior que ahora empieza su camino, heredará bastantes rasgos de los MOOC's, pero será un producto híbrido con pluralidad de opciones metodológicas donde el parámetro ayuda pedagógica tendrá valores que oscilen entre el MOOC puro, a una individualización basada en el perfil de aprendizaje y en la analítica que se haga para cada caso. 
Como hemos visto la aparición de los MOOC’s se sitúa a caballo entre dos épocas donde la configuración social, cultural, humana, de las sociedades cambia (de la era industrial a la era del conocimiento) y dos etapas muy diferenciadas del desarrollo de las teorías del aprendizaje: La primera donde las teorías y la práctica de la organización educativa están orientadas para describir y clasificar a los alumnos desde la perspectiva de sus capacidades, y la segunda caracterizada por las teorías orientadas a maximizar el aprendizaje de todos los alumnos. Con distintos valores: en unas el progreso se mide en función del tiempo (de pautas y ciclos temporales: cursos, clases, etc.), en otras se basa en los logros individuales (Reigeluth, 2012).

La aparición de los MOOC’s, en una línea continua desde los recursos educativos abiertos y el acceso abierto a los fondos de la producción científica, pone al alcance de cualquier persona en cualquier lugar los mismos contenidos que disponen las universidades más prestigiosas, y los más reconocidos especialistas, para la formación reglada y para la obtención de títulos.

Tres son las razones esgrimidas por Roscorla (2012) para que se utilice los servicios de esta tecnología, a saber: para acceder a cursos de alta calidad; para experimentar; para lograr mayor independencia.

\section{MODELO DE NEGOCIO Y CRÍTICAS A LOS MOOC}

Las primeras respuestas giraron en torno al pago de pequeñas cantidades (entre 30 y 80 dólares) por la expedición de certificados de asistencia (badges) una vez concluido el curso. Idea que ha ido enriqueciéndose con propuestas de acreditación con modelos parecidos a los certificados profesionales, con agentes que puedan actuar como "certificadores externos" de las competencias adquiridas. Es decir, se trata de un pago una vez superado el curso. El punto débil de este primer modelo serían las altas tasas de abandono cercanas al 80\%-90\%.

Otra propuesta de modelo más interesante sería la de convertirse directamente en una agencia de colocación. Quién mejor que la propia universidad y el profesorado para conocer perfectamente no sólo los conocimientos adquiridos por el candidato sino (gracias a los nuevos sistemas de analítica de aprendizaje y a los nuevos sistemas de acreditación basados en la obtención de badges y karmas) también cuáles son las habilidades soft del candidato, cómo trabaja en grupo, cuáles son sus capacidades de liderazgo e improvisación, de capacidad respuesta a la incertidumbre y de comunicación.

Una idea especialmente interesante vinculada a su papel como head hunters es que sea la comisión cobrada a las empresas por la identificación y colocación de los alumnos brillantes (en torno al 5\%) lo que asegure la sostenibilidad del modelo. Que sean los alumnos más brillantes los que aseguren la formación gratuita del resto. Poco a poco han ido surgiendo otras propuestas de negocio como cobrar por servicios extras como tutorías, acceso a recursos extras, sesiones de networking. El debate sobre los modelos de negocio que pueden estar detrás de los MOOC's sigue de máxima actualidad. 
En los últimos meses, mientras la ola de los MOOC's subía y subía en los medios de comunicación y en las redes sociales, hemos visto aparecer cada vez con más fuerza las voces críticas.

Una acertada definición de MOOC la podemos hacer a partir de las apreciaciones de Wiley (2012): "MOOC es un término que viola todas sus letras menos una” ya que muchos son enormes pero no están abiertos (ejemplo: http://www.udacity.com/legal/), muchos están abiertos pero no son masivos (ejemplo: http://learninganalytics.net/syllabus.html), muchos se esfuerzan por no ser cursos, no tienen elementos esenciales como es la evaluación (por ejemplo, http://cck11.mooc.ca/how.htm). Al menos todos hasta la fecha han sido en línea. Tampoco son abiertos ni gratuitos, por ejemplo eCornell ya ofrece un MOOC al que únicamente pueden acceder estudiantes que lo cursen pagando (Mangan, K. 2013).

Pero sobre todo, la crítica más recurrente es el caos existente, ya que se denominan MOOC's a un totum revolutum existente cuando realmente corresponden a dos grupos diferentes. Los cMOOC's que están más orientados a un aprendizaje conectividad y que por tanto fomentan el aprendizaje participativo y basado en comunidades, mientras que los XMOOC's son cursos cerrados, basados exclusivamente en contenidos y alejados de la tesis conectivista de que el aprendizaje es efectivo cuando se realiza en comunidad.

Son muchas las voces que proclaman un exceso de despliegue publicitario de los MOOC's, y todo parece indicar que se está de lleno en la curva descendente de la hipérbola, empujados por voces críticas en torno a cuestiones metodológicas, a su calidad, a las posibles formas de evaluación, a su modelo de negocio, a su pertinencia o no para todo tipo de institución. Críticas que señalan como algo decepcionante desde el punto de vista de la innovación metodológica volver al aprendizaje a través casi exclusivamente de clases magistrales (vídeos enlatados). Voces que ven los MOOC’s como una vuelta atrás metodológica no justificada por el hecho de llegar a más alumnos y a precios gratuitos. El tema es de tal calado y tiene tantas implicaciones que no son pocas las iniciativas que están intentando entender el fenómeno MOOC en toda su profundidad y riqueza.

Algunas de estas iniciativas son: MOOC sobre MOOC’s², MOOC’s sobre educación $^{3}$ o, sobre otras etapas educativas, Moocificación ${ }^{4}$.

Una pequeña consulta, no representativa desde el punto de vista estadístico, a profesores universitarios de diferentes universidades españolas, identifica las siguientes críticas a los MOOC's:

1. La educación requiere un diálogo.

2. El aprendizaje auténtico no es escalable, no se puede multiplicar.

\footnotetext{
${ }^{2}$ http://www.moocmooc.com/

${ }^{3}$ http://openeducacion.us

${ }^{4}$ http://www.hybridpedagogy.com/Journal/files/MOOC_Pedagogy.html
} 
3. Más apoyo, no menos, equivale a una calidad educativa mejor.

4. Los MOOC's van acompañados de costes importantes.

5. Los MOOC's se deberían alinear con las metas estratégicas.

6. El peligro de los MOOC's como un sustituto para ahorrar dinero.

Además es muy interesante el determinar cómo van a ser evaluados los conocimientos adquiridos por medio de este nuevo método de aprendizaje. Sin lugar a dudas es una cuestión complicada de tratar ¿cómo se puede evaluar el aprendizaje de un curso en el que participan más de 100.000 alumnos? ¿Cuántos profesores hacen falta?

La solución más común a este problema es emplear un sistema llamado per assessment en el que los propios alumnos se califican entre ellos mismos, siendo necesaria la calificación de otros alumnos para mejorar la propia. Además, con el fin de lograr una mayor fiabilidad, un ejercicio de un alumno no es evaluado por un único alumno, sino por varios, y se puede emplear un sistema estadístico para detectar posibles discrepancias en la evaluación realizada. Si bien se está imponiendo el sistema de evaluación por rúbrica definido por Vera Vélez (2008) como "un instrumento de medición que tiene criterios establecidos y estándares de desempeño por niveles y escalas, con el propósito de determinar la calidad de la ejecución de tareas específicas del estudiante”.

\section{MOOC Y BIBLIOTECAS DE INVESTIGACIÓN}

Cuando sea posible, las bibliotecas de investigación deben participar en conversaciones en torno a MOOC's y promover sus valores fundamentales. Porque al hacerlo, también fomentará la continua vitalidad de las bibliotecas como socios en el desarrollo Alfin y enriquecer además su lifestream. La adaptación a estas nuevas demandas es sin duda un reto para las bibliotecas de investigación, de lo contrario se verán abocadas a la exclusión del universo MOOC.

Las bibliotecas de investigación han de asumir nuevas versiones de viejas responsabilidades y apoyar este nuevo modo de enseñar y aprender. Hasta ahora pueden vislumbrarse tres vertientes que están llevando las bibliotecas de investigación con esta nueva corriente:

- Están cumpliendo con labores de diseminación y apoyo a los cursos MOOC impartidos por sus propios campus.

- Las bibliotecas de investigación se han encargado de trabajar con profesores y departamentos para afrontar el nuevo ecosistema creado por los derechos de autor planteadas por la enseñanza en el entorno abierto y en línea.

- Las bibliotecas de investigación se convierten en centros selectivos de conocimiento para ayudar a los profesores a identificar y localizar los materiales alternativos que están libres de restricciones de derechos de autor, ya sea debido a que son de dominio público o porque están disponibles bajo licencia Creative Commons. 
Además de este control de información y de derechos de autor derivado de toda la problemática de la disponibilidad y acceso a contenidos externos, la biblioteca también deberá estar capacitada para poner a disposición de los alumnos cuanta información se estime oportuna y relevante para su función de acompañamiento en las labores de formación. Además los MOOC’s, aunque originariamente no están diseñadas ni preparadas para eso, sí que tendrán que estar muy atentas a realizar también labores de acompañamiento y asesoramiento en las tareas de investigaciones inherentes a la universidad, ya que los MOOC's podrán convertirse en plataformas para facilitar formación reglada virtual y por lo tanto también con la necesidad de realizar trabajos fin de grado, fin de máster, y quién sabe si investigaciones en el ámbito de doctorado. Evidentemente es muy aventurado el pensar que labores de investigación vayan a realizarse por medio de los MOOC's, no obstante existe una tendencia clara a la realización de formación en línea por parte de prácticamente todas las instituciones de enseñanza superior.

\section{MOOC EN LA UNIVERSIDAD ESPAÑOLA}

Son aún escasas las posibilidades que tenemos de realizar cursos por medio de MOOC’s en las universidades españolas o latinoamericanas, pero esta realidad está cambiando de forma rápida. La sensibilidad hacia este nuevo modelo de negocios es cada vez mayor, especialmente derivado por la precaria situación del sistema universitario español el cual está condenado a mutar por ajustes severos en su presupuesto y recursos humanos, y especialmente por lo que se prevé como un cambio legislativo histórico y demoledor de las raíces y tradiciones de la universidad española.

Ante un panorama incierto, las universidades intentan reinventarse continuamente para cazar y atraer a alumnos. Este modelo está siendo adoptado tanto por universidades públicas como privadas.

En la plataforma Miriada X pueden observarse los cursos que facilitan dos instituciones universitarias no españolas pero que sí se desean destacar al ser de habla hispana: La Universidad Abierta para Adultos de la República Dominicana y la Universidad de Puerto Rico, el National University College.

De las muchas universidades inscritas en la página del Ministerio de Educación del Gobierno de España (más de 80 universidades) no llegan a veinte las universidades que facilitan formación por medio de MOOC's.

Prácticamente todas utilizan la plataforma Miríada X, la cual es utilizada por las universidades inscritas en la Red Universia, siendo accesible para todos de manera gratuita.

Algunas de las Universidades que actualmente están realizando formación a través de los MOOC's son: Universidad Nacional de Educación a Distancia, Universidad de Cantabria, Universidad de Murcia, Universidad de Huelva, Universitat de Girona, Universidad de Alcalá, Universidad Politécnica de Valencia, etc.

Las temáticas de estos cursos es muy variada temáticamente aunque la oferta formativa no es muy extensa, no obstante repetimos que el crecimiento de oferta es actualmente exponencial y son cada vez más los profesores implicados en impartir 
formación por vías alternativas diferentes a la presencialidad tradicional. Aún no está tan claramente implantada en España como en otros ámbitos geográficos, no obstante parece imparable el proceso de expansión por la universidad española.

\section{CONCLUSIONES}

La reciente aparición de MOOC's en el ámbito universitario ha sido una sorpresa para mucho de sus gestores y también para el profesorado que siempre ha pecado de falta de adaptación a los medios formativos. No obstante, la adaptación y el mimetismo con las fórmulas y necesidades es cada vez mayor, derivado de la crisis universitaria que queda plasmada en el número de alumnos y la continua adaptación a modelos anglosajones donde la internacionalización y la virtualización formativa se está potenciado de manera diáfana. Los MOOC's son de reciente aparición, no obstante es destacable señalar su rápido desarrollo, su crecimiento exponencial, y la vinculación que rápidamente han tenido con estos métodos universidades de prestigio mundial.

Los MOOC's potencian la presencia de las universidades ante la sociedad, ante los potenciales alumnos-clientes del futuro, muestra la valía del profesorado y contenidos facilitados. La universidad lo utiliza como forma de marketing pero también como método de financiación desde el momento que la formación sí es gratuita, así como el acceso a los contenidos, no así la expedición de títulos o certificados que avalan la formación.

El modelo de negocio es evidente, la tecnología está al alcance de todos, la oferta formativa cada vez es mayor, la sensibilización de las comunidad universitaria un hecho, por lo que parece claro que los MOOC's no son un fenómeno esporádico, sino que parece que el modelo ha venido para quedarse en nuestra sociedad, por lo que las universidades deberán ver como engarza estos nuevos métodos con la visión, misión y filosofía de cada universidad, y por supuesto a tenor de los continuos cambios legislativos que sufre el sistema universitario español.

\section{BIBLIOGRAFÍA}

DOWNES, Stephen (2008). Mooc and Mookies: the connectivism \& connective knowledge online course

http://www.slideshare.net/Downes/mooc-and-mookiesthe-connectivismconnective-knowledge-online-course-presentation?type=powerpoint

DOWNES, Stephen (2011). Free Learning. Essays on open educational resources and copyright

http://www.downes.ca/files/books/FreeLearning.pdf

HARVARD BUSINESS REVIEW (2013). Disruptive trends to watch in 2013. http://blogs.hbr.org/anthony/2013/01/disruptive_trends_to_watch_in.html

MANGAN, Katherine (2013). eCornell Offers a MOOC That Steers Students to a Paid Follow-Up. The Cronicle Higher Education 
http://chronicle.com/blogs/wiredcampus/ecornell-to-offer-mooc-that-steersstudents-to-for-credit-follow-up/41433

THE NEW YORK TIMES. SCIENCE (2011). Virtual and artificial, but 58.000 want course.

http://www.nytimes.com/2011/08/16/science/16stanford.html

REIGELUTH, C.M. (2012). Teoría instruccional y tecnología para el nuevo paradigma de la educación. http://www.um.es/ead/red/32/reigeluth_es.pdf

ROSCORLA, Tanya (2012). 3 reasons why people take massively open online course. http://www.centerdigitaled.com/policy/Why-Massively-Open-OnlineCourses.html

SIEMENS, G. (2005), Conectivismo: Una teoría del aprendizaje para la era digital. http://es.scribd.com/doc/201419/Conectivismo-una-teoria-del-aprendizaje-para-laera-digital

THRUN, S.; NORVIN, P (2012). Online introduction to Artificial Intelligence. https://www.ai-class.com/

VERA VÉLEZ, L. (2008). La rúbrica y la lista de cotejo. http://www.tecnoedu.net/lecturas/materiales/lectura10.pdf

WIKIPEDIA (2013). MOOC. http://es.wikipedia.org/wiki/MOOC

WIKIPEDIA (b) (2013). Cultura libre. http://es.wikipedia.org/wiki/Cultura_libre

WIKIPEDIA (c) (2013). Conectivismo. http://es.wikipedia.org/wiki/Conectivismo 